\title{
Theoretical Study of Dielectric Properties in Triglycine Sulphate Crystal
}

\author{
Naveen Kohli ${ }^{*} \cdot$ Trilok Chandra Upadhyay $^{1}$ \\ ${ }^{1}$ Department of Physics, Govt. PG College Joshimath (Sri Dev Suman University Uttarakhand), India \\ *Corresponding Author Email Id: navkpguk1001@gmail.com
}

Received: 2.8.2021; Revised: 19.10.2021; Accepted: 21.10.2021

CSociety for Himalayan Action Research and Development

\begin{abstract}
Present paper investigate theoretically dielectric properties such as dielectric constant, soft mode frequency and tangent loss by considering two sub lattice pseudospin, spin-lattice coupled mode model (PLCM) and adding third and fourth-order anharmonic (phonon) and spin-lattice interaction terms and using thermal Green's function (double time) method expression for dielectric constant, soft mode frequency, and tangent loss is derived for TGS crystal. Model value have been fitted in expression and their temperature dependence have been calculated for TGS crystal. Theoretical results have been correlated with experimental results of (Aravzhi et al 1997), which is approximate close to experimental results.
\end{abstract}

Keywords- Frroelectric, Delectric constant, Phonon, Anharmonic interaction

\section{Introduction}

TGS is a type of order-disorder ferroelectrics with chemical formula $\left(\mathrm{NH}_{2} \mathrm{CH}_{2} \mathrm{COOH}\right)_{3} \mathrm{H}_{2} \mathrm{SO}_{4}$. It has wide range of application in the field of optoelectronic mainly in infrared detectors, capacitors, transducer, optical transmitters etc(Sun et al.1999,Krajewski et al.1980). The crystal have low dielectric constant and large pyroelectric coefficient. It is H-bonded crystal and show second-order phase transition at Curie temperature $322 \mathrm{~K}$ (Bharthsarathi T., et al.,2010). It is monoclinic in ferroelectric as well as in paraelectric phases. The lattice parameter at room temperature for this crystal are $a=9.15 \AA, b=12.69 \AA, c=5.73 \AA, \beta$ $=105.4^{0}$ at higher temperature this parameter change $\mathrm{a}=9.320 \AA, \mathrm{b}=7.277 \AA, \mathrm{c}=8.970 \AA, \beta=114.91^{0}$.

A variety of work on TGS crystal have been carried out by many experimentalist. Crystal structure of this crystal are first studied by Hoshino (Hoshino et al.1959) and revealed the essential ferroelectric parameter. Ostrowski et al.,(2012) studied the dielectric properties of fine grained triglycine sulphate and observed shift in $\mathrm{T}_{c}$, permittivity by reducing the particles size and application of hydrostatic pressure. have done synthesis, growth and electrical transport properties studies on pure $\mathrm{LiSO}_{4}$ doped TGS and pure crystals properties, such as growth, synthesis and transport(electrical) were studied (Khanum and Podder, 2011). Size-driven ferroelectric transition in TGS nano composite studied by Cizman et al,(2013). Optical, structural study of KDP-doped TGS crystal is carried out by Deepti and Shanti(2014). Prasolve at al(1998) studied dielectric hysteresis loop of TGS crystal. Yamaguchi et al(2006) carried out dilatometric study on TGS crystal. Alexandru et al have made dielectric relaxation study on TGS crystal. 
Theoretical studied on TGS crystal first done by using Ising model (Blinc et al,1961). Later on (Tello and Harnandez, 1973) studied transition mechanism of TGS using tunneling model. A Vibrational study of phase transition in TGS crystal is done by (Chaudhuri et al,1988) by considering two-sub lattice model(pseudo spin). They considered Green's function method (Zubarev,1960) and find the relation for soft mode frequency, susceptibility, dielectric constant and transition temperature. They (Chaudhuri et al,1988) have not taken third order anharmonic (phonon) and spin lattice interaction terms. They could not find better and decisive results as they disassociate the correlation in beginning.

In this work we considered, third, fourth order anharmonic and spin lattice interaction terms in Two-SubLattice model (PLCM). We use Green's Function (Double Time) method and simplifying PLCM model to derive the relation for width, shifts, dielectric constant, tangent loss and soft mode frequency. The model value of various physical quantities are fitted in derived relation, hence their temperature variation are examined theoretically. The Theoretically obtained values for these relation (soft mode frequency, tangent loss and dielectric constant) is compared with experimental results for TGS crystals (Aravazhi et al,1997)

\section{Hamiltonian Formalism}

The two sub-lattice pseudo spin- lattice coupled mode model (PLCM) for TGS crystal is taken as

$$
\begin{aligned}
& H_{a}=-2 \Omega \sum_{i}\left(S_{1 i}^{X}+S_{2 i}^{X}\right)-\sum_{i j}\left[J_{i j}\left(S_{1 i}^{Z} S_{1 j}^{Z}+S_{2 i}^{Z} S_{2 j}^{Z}\right)+K_{i j} S_{1 i}^{Z} S_{2 j}^{Z}\right] \\
& -\sum_{k} V_{i k}\left(S_{1 i}^{Z} A_{k}+S_{2 j}^{Z} A_{k}^{+}\right)+\frac{1}{4} \sum_{k} \omega_{k}\left(A_{k}^{+} A_{k}+B_{k}^{+} B_{k}\right)
\end{aligned}
$$

Here $s_{\alpha}^{m}(\mathrm{~m}=\mathrm{x}, \mathrm{z})$ is Pseudo spin variable's(S) component, $\Omega$ - tunneling frequency(proton), $\mathrm{J}_{\mathrm{ij}}$ and $\mathrm{K}_{\mathrm{ij}}$ are coupling constants of coupling of same and different lattices. $\mathrm{V}_{\mathrm{ik}}$ is constant corresponding to lattice-spin interaction, $\omega_{\mathrm{k}}$ represent frequency(phonon), $\mathrm{A}_{\mathrm{k}}$ is position and $\mathrm{B}_{\mathrm{k}}$ momentum operator.

In Eq.(1) we add third, fourth order anharmonic(phonon) interactions terms

$$
H_{b}=\sum_{k_{1} k_{2} k_{3}} V^{(3)}\left(K_{1}, K_{2}, K_{3}\right) A_{k_{1}} A_{k_{2}} A_{k_{3}}+\sum_{k_{1} k_{2} k_{3} k_{4}} V^{(4)}\left(K_{1}, K_{2}, K_{3}, K_{4}\right) A_{k_{1}} A_{k_{2}} A_{k_{3}} A_{k_{4}},
$$

where first and second term in eq.(2) and are third, fourth order force constants respectively.

we shall add

$$
H_{c}=-2 \mu E \sum_{i}\left(S_{1 i}^{Z}+S_{2 i}^{Z}\right)-\sum_{i k} V_{i k}\left(S_{1 i}^{X} A_{k}+S_{2 i}^{X} A_{k}^{+}\right)
$$

where $\mu$ is dipole moment of $\mathrm{O}-\mathrm{H}---\mathrm{O}$ bond and $\mathrm{E}$ external electric field. The first term describes effect of external electric field on crystal and the second term reveal an indirect coupling in tunneling movement of two proton between two equilibrium position of $\mathrm{O}-\mathrm{H}---\mathrm{O}$ bonds. 
the total Hamiltonian is

$$
H_{\text {tot. }}=H_{a}+H_{b}+H_{c}
$$

\section{Method}

From D.N. Zubarev(1960), we introduced Green's function

$$
\begin{gathered}
G_{i j}\left(t-t^{\prime}\right)=\left\langle\left\langle S_{1 i}^{z}(t) ; S_{1 j}^{z}\left(t^{\prime}\right)\right\rangle\right\rangle, \\
=-i \theta\left(t-t^{\prime}\right)\left\langle\left[S_{1 i}^{z}(t) ; S_{1 j}^{z}\left(t^{\prime}\right)\right]\right\rangle
\end{gathered}
$$

In Eq. (4) $S_{1 i}^{z}$ is spin variable, $\theta$ is step function, $\theta=0(\mathrm{t}<\mathrm{t}) \& \theta=1(\mathrm{t}>\grave{\mathrm{t}})$. Differentiating Eq. (4) two times w.r.t. $\mathrm{t} \& \mathrm{t}$ respectively, find Fourier transform and put in Dyson's equation form we obtain

$$
G_{i j}(\omega)=G_{I J}^{0}(\omega)+G_{i j}^{0}(\omega) \tilde{P}(\omega) G_{i j}^{0}(\omega)
$$

Where $G_{i j}^{0}(\omega)$ is Green's function in unperturbed form, and $\tilde{P}(\omega)$ is operator corresponding to polarization. These are given as

$$
\begin{gathered}
G_{i j}^{0}(\omega)=\frac{\Omega\left\langle S_{1 i}^{x}\right\rangle \delta_{i j}}{\pi\left[\omega^{2}-4 \Omega^{2}\right]}, \\
\tilde{P}(\omega)=\pi i \frac{\left\langle\left[F_{1 i} ; S_{1 j}^{y}\right]\right\rangle}{\Omega\left\langle S_{1 i}^{x}\right\rangle^{2}}+\frac{\pi^{2}}{\Omega^{2}\left\langle S_{1 i}^{x}\right\rangle^{2}}\left\langle\left\langle F_{1 i} ; F_{1 j}^{\prime}\right\rangle\right\rangle
\end{gathered}
$$

where

$F(t)=2 \Omega V_{i k} A_{k} S_{1 i}^{z} \delta_{i j}-2 \Omega J_{i j}\left(S_{1 i}^{z} S_{1 i}^{x} \delta_{i j}+S_{1 i}^{x} S_{1 j}^{z}\right)-V_{i k} A_{k} J_{i j}\left(S_{1 i}^{z} S_{1 i}^{x} \delta_{i j}+S_{1 i}^{x} S_{1 i}^{z}\right)-2 \Omega K_{i j} S_{1 i}^{x} S_{2 j}^{z}$

The second part of $\tilde{P}(\omega)$ have higher order Green functions $\left\langle\left\langle F_{1 i} ; F_{1 j}^{\prime}\right\rangle\right\rangle$, which are like $<<\mathrm{mn}, \mathrm{pq}>>$, $<<$ mnp,qrs>>. These can be disassociate into simpler ones and then solved. In this way $\tilde{P}(\omega)$ is evaluated. Eq. (5) gives Green's function finally as

$$
G_{i j}(\omega)=\frac{\Omega\left\langle S_{i}^{x}\right\rangle \delta_{i j}}{\pi\left[\omega^{2}-\tilde{\Omega}^{2}-\tilde{P}(\omega)\right]}
$$

where

$$
\begin{aligned}
& \widetilde{\Omega}^{2}=a^{2}+b^{2}-b c \\
& a=2 J_{0}\left\langle S_{1}^{z}\right\rangle+K_{0}\left\langle S_{2}^{z}\right\rangle+2 \mu E
\end{aligned}
$$




$$
b=2 \Omega
$$

$$
c=2 J_{0}\left\langle S_{1}^{z}\right\rangle+K_{0}\left\langle S_{2}^{x}\right\rangle
$$
obtain

Putting the value of $\tilde{P}(\omega)$ into Eq. (9) and resolving into real $\Delta(\omega)$ and imaginary $\Gamma(\omega)$ parts we

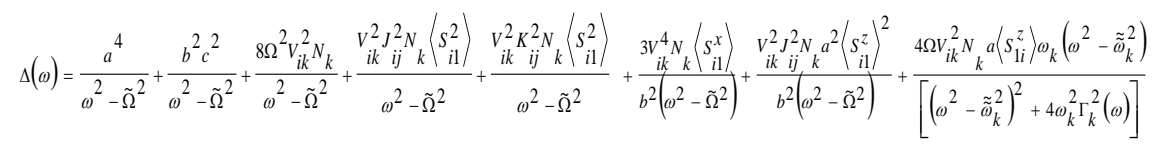

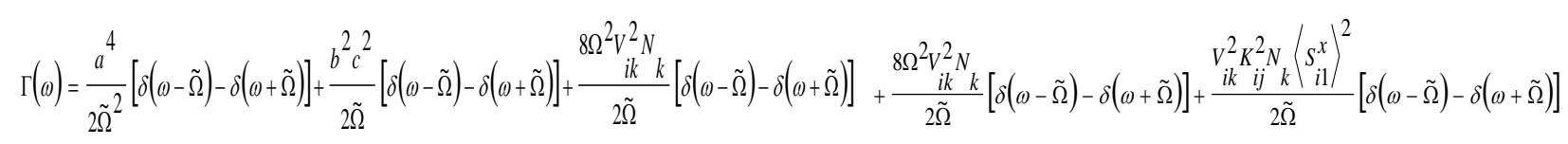

$$
\begin{aligned}
& +\frac{V_{i k}^{4} N a^{2}}{2 \tilde{\Omega} b^{2}}[\delta(\omega-\tilde{\Omega})-\delta(\omega+\tilde{\Omega})]+\frac{4 \mu^{2} E^{2} V_{i k}^{2} N_{k} a^{2}}{2 \tilde{\Omega} b^{2}}[\delta(\omega-\tilde{\Omega})-\delta(\omega+\tilde{\Omega})]+\frac{8 \Omega V_{i k}^{2} \omega_{k}^{2} \Gamma_{k}(\omega)\left(\left\langle s_{1 i}^{x}\right\rangle+\left\langle s_{1 i}^{z}\right\rangle\right)}{2\left[\left(\omega^{2}-\widetilde{\omega}_{k}^{2}\right)^{2}+4 \omega_{k}^{2} \Gamma_{k}^{2}(\omega)\right]}
\end{aligned}
$$

In Eq. (14) \& (15) $\tilde{\widetilde{\omega}}_{k}$ is phonon frequency (modified), $\Delta_{k}(\omega)_{\text {is phonon shift, }} \Gamma_{k}(\omega)$ is phonon width. They can be derived by solving Green's function(phonon) $\left\langle\left\langle A_{k} ; A_{k^{\prime}}^{+}\right\rangle\right\rangle$\& by using phonon Hamiltonian only.

With the help of shifts $\Delta(\omega)$ one can analyse variation in the value of frequency i.e shifts in peak of frequency curve. The width $\Gamma(\omega)$ describe width between two point which is half the maximum of the frequency curve.

\section{Ferroelectric (Soft) Mode Frequency}

Now putting the above values of $\mathrm{P}(\omega)$ into Eq. (9) finally, the Green function becomes

$$
G_{i j}(\omega)=\frac{\Omega\left\langle S_{i}^{x}\right\rangle \delta_{i j}}{\pi\left[\omega^{2}-\widetilde{\Omega}^{2}+2 i \Omega \Gamma(\omega)\right]}
$$

Where

$$
\begin{aligned}
& \hat{\Omega}^{2}=\widetilde{\widetilde{\Omega}}^{2}+\Delta_{s-p}(\omega) \\
& \widetilde{\widetilde{\Omega}}^{2}=\widetilde{\Omega}^{2}+\Delta_{s}(\omega)
\end{aligned}
$$

After solving we obtain expression as

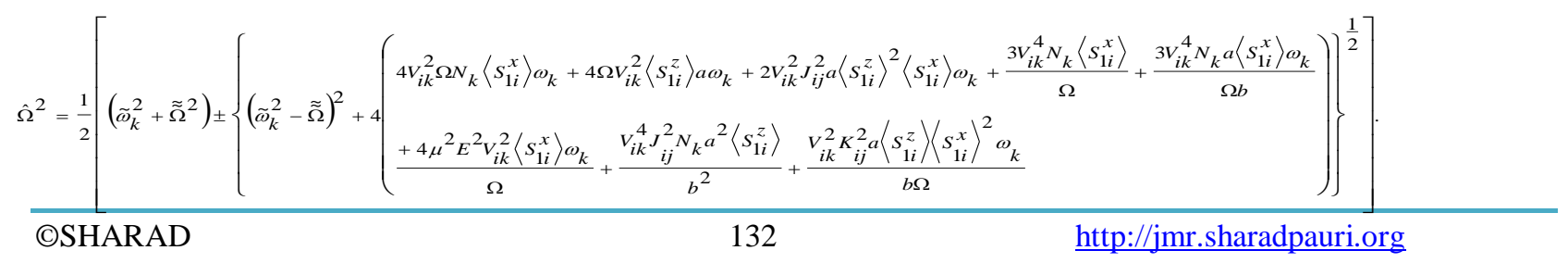


The frequency $\hat{\Omega}$ is soft mode frequency of TGS crystal. It is clear from above equation that temperature depended various interaction terms have fine impact on soft mode which contains third, fourth- order anharmonic interaction, electric field and extra spin lattice interaction terms.

\section{Dielectric Constant, Tangent Loss}

The effect of electric field in ferroelectric or dielectric crystal is expressed by susceptibility. This is related to Green's function as

$$
\chi=-\lim _{x \rightarrow 0} 2 \pi \chi N \mu^{2} G_{i j}(\omega+i x)
$$

Here $\mathrm{N}$ is dipoles no. in unit cell and $\mu$ is dipole moment in crystal. The relation between dielectric constant $\varepsilon$ and susceptibility $\chi$ is

$$
\varepsilon=1+4 \pi \chi
$$

For ferroelectric crystals $\varepsilon>>1$

$$
\varepsilon=4 \pi \chi
$$

From Eq. (18), (22) and (23) we find expression for dielectric constant as

$$
\varepsilon(\omega)=\frac{8 \pi N \mu^{2} \Omega\left\langle S_{1 i}^{x}\right\rangle \delta_{i j}\left(\omega^{2}-\hat{\Omega}^{2}\right)}{\left[\left(\omega^{2}-\hat{\Omega}^{2}\right)^{2}+2 \Omega^{2} i \Gamma^{2}(\omega)\right]}
$$

Where $\varepsilon(\omega)>>1$. Eq. (23) shows that dielectric constant is inversely proportional to soft mode frequency $\hat{\Omega}$, width $\Gamma(\omega)$ and directly to tunneling frequency $\Omega$ of proton. However in numerical calculation dielectric constant we neglect phonon frequency $\omega$ from numerator and denominator also width term from denominator as their values are extremely small. Therefore finally dielectric constant are expressed as

$$
\varepsilon(\omega)=\frac{8 \pi N \mu^{2} \Omega\left\langle S_{1 i}^{x}\right\rangle}{\hat{\Omega}^{2}}
$$

The power dissipated in dielectric crystal is taken as loss tangent $(\tan \delta)$ and can be express as proportion of imaginary $\left(\varepsilon^{\prime \prime}\right)$ and real $(\varepsilon)$ part of dielectric constant $(\varepsilon)$

$$
\tan \delta=\frac{\varepsilon^{\prime \prime}}{\varepsilon^{\prime}}=-\frac{2 \Omega \Gamma(\omega)}{\omega^{2}-\hat{\Omega}^{2}}
$$


Eq.. (24) and Eq. (25) reveal that dielectric constant and tangent loss is depended on modified soft mode frequency. Hence, also depended on tunneling frequency and anharmonic interaction terms.

\begin{tabular}{lcccccccc}
\hline $\begin{array}{l}\text { Physical } \\
\text { Parameters } \\
\text { Values }\end{array}$ & $\omega_{k}^{2}$ & $\Omega$ & $J$ & $K$ & $V_{i k}$ & $T_{c}$ & $N_{\mu}$ & $A_{k}$ \\
& 0.59 & $\left.\begin{array}{c}0.10\left(\mathrm{~cm}^{-}\right. \\
1\end{array}\right)$ & $340\left(\mathrm{~cm}^{-1}\right)$ & $170\left(\mathrm{~cm}^{-1}\right)$ & $10\left(\mathrm{~cm}^{-3 / 2}\right)$ & $322(\mathrm{~K})$ & $10^{18} \mathrm{esu}$ & 10.2 \\
\hline
\end{tabular}

Table 1. Physical parameters for TGS crystal (Chaudhuri et al.,1988)

\section{Results}

With the help of various physical parameters taken from literature and fitting it in obtained expression for dielectric $\operatorname{constant}(\varepsilon)$, tangent loss $(\tan \delta)$ and soft mode frequency $(\hat{\Omega})$, their thermal variation have been plotted(Fig. 1-3) for TGS ferroelectric crystal. It is seen from plot (fig.1,2), the dielectric constant and tangent loss increases with temperature and become maximum at $T_{c}$ and then decreases. Soft mode frequency decreases first until ceases (softening) at $\mathrm{T}_{\mathrm{c}}$ and further increases with temperature (fig.3).

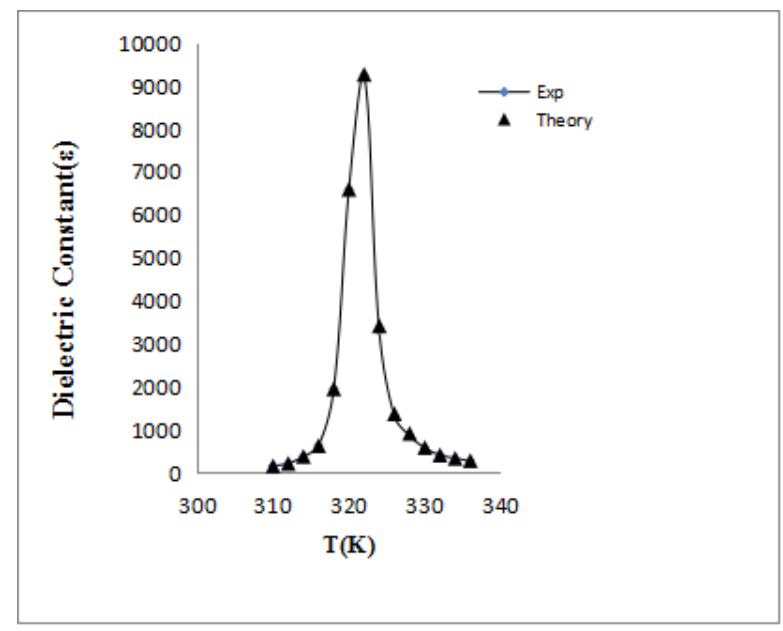

Figure 1 - Thermal dependence of dielectric constant in TGS Crystal. 


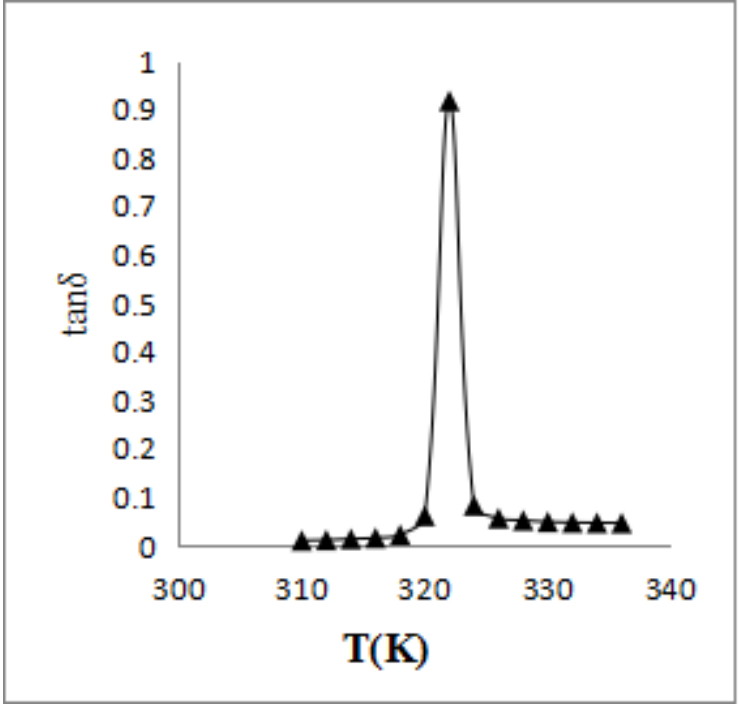

Figure 2- Thermal dependence of tangent loss $(\tan \delta)$ in TGS crystal

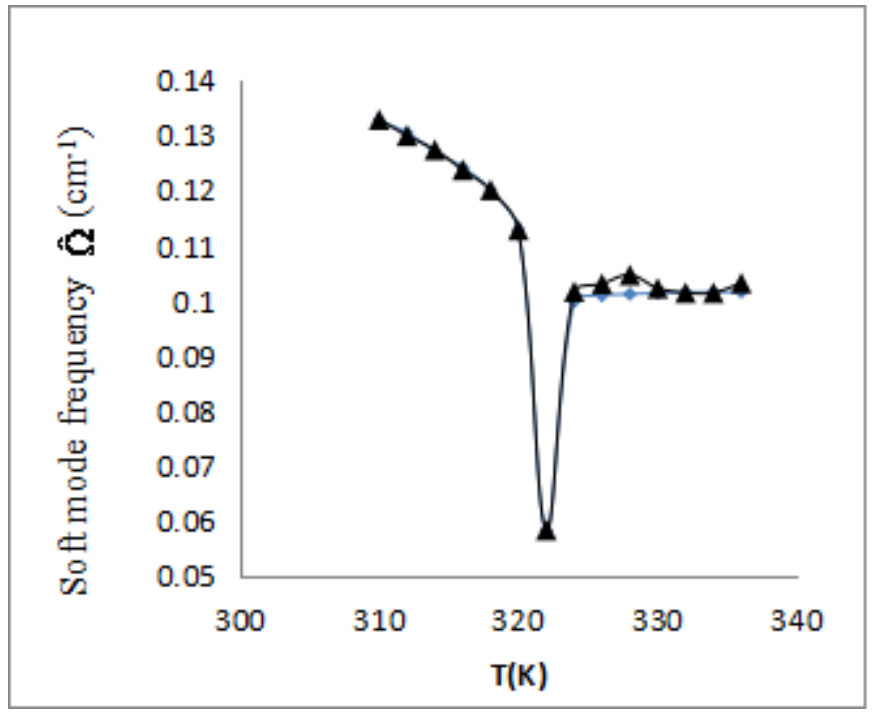

Figure 3- Thermal dependence of soft mode frequency in TGS Crystal

\section{Discussion and Conclusion}

In Present work, we modify two sub-lattice pseudo spin lattice coupled mode model (PLCM) by extending earlier work of (Chaudhury etal,1988) In this model we considered third, fourth order anharmonic(phonon), spin-lattice interaction term and electric field terms. However, in numerical calculation we take electric field term zero to make calculation simple. We disassociate the correlation at proper stage. With the help of simplified model and differentiating Green's Function two time (double time Green's functions method (Zubarev,1960) the expression for dielectric susceptibility and hence dielectric $\operatorname{constant}(\varepsilon)$ and tangent $\operatorname{loss}(\tan \delta)$ have been obtained in this paper. Using model values (Table 1) of quantities in expression for dielectric constant $(\varepsilon)$ and tangent loss, numerically temperature dependences of these have been calculated for Triglycine Sulphate (TGS),. Theoretically results have been compared with experimental reported by (Aravazhi etal,1997). Our results approximately agree with experimental results of other well. Expression of dielectric constant reveals that, the dielectric constant

depends on soft mode frequency $\hat{\Omega}$ and it is inverse relation to square of soft mode frequency $\hat{\Omega}$. As soft mode frequency contains the effects of extra-spin lattice interactions terms, also of phonon anharmonic terms. This shows that dielectric constant depends on extra-spin lattice interactions terms in addition to phonon-phonon anharmonic interaction terms. The dielectric constant $(\varepsilon)$ increases at Tc because soft mode frequency $\hat{\Omega}$ decreases near Tc.

The expression for loss tangent $(\tan \delta)$ show that it is also depended on tunneling frequency $(\Omega)$, soft mode frequency $\hat{\Omega}$ and total width $\Gamma(\omega)$. The values of tangent loss increase and become maximum near 
Transition temperature. Due to high domain mobility near transition temperature dielectric losses increase near transition temperature.

Therefore, it is concluded that the modified model explains better quantitatively the dielectric properties of TGS, crystals. This simplified model can be applied for the study of similar other ferroelectric, antiferroelectric H-bonded crystals.

\section{Acknowledgements}

Authors are grateful to whole Physics Department HNBGU for their kind encouragements and for their valuable help.

\section{References}

Aravazhi,Jeyavel S, Subramanian R,(1997), Growth and Characterization of benzophenone and urea doped triglycine sulphate crystal Ferroelectrics. 200:279-286.

Bharthsarathi T,O.P.Thakur,Murugakoothan P,(2010), Studies on the effectof L-Glutamine on morphology. Structure,optical, mechanical and electrical properties of TGS crystal Physica B 405: 3943-3948

Blinc R, Detoni S and Pintar M(1961), Nature of ferroelectric transition inTriglycine Sulphate Phys Rev, 124:1036.

Chaudhuri B.K,. Chaudhuri K.R \& Banerjee S(1988), Green Function Theory of ferroelectric phase transition in Hydrogen-bonded Triglycine sulphate with pseudospin - lattice coupled mode model Phys. Rev. B,38(1):689-707.

Cizman A, Antropova T, Poprawski R,(2013),Size- Driven ferroelectric-paraelectric phase transition in TGS nanocomposites, Journal of Nanoparticles Research 15:1807

Deepti and Shanti(2014), Structural and Optical studies of potential ferroelectric crystal KDP Doped TGS, J Scientific Res, 6(1): 1

Hoshino,Okaya S, Pepinsky Y, (1959), Crystal structure of ferroelectric phase of (Glycine) ${ }_{3} \mathrm{H}_{2} \mathrm{SO}_{4}$ Phys. Rev. 115(2): 323.

Khanum and J. Podder(2011), Structural and optical properties of Triglycine Sulphate Single crystal doped with Potassium Bromide, J Crystallization Processes and Tech,1(2P),15

Krajewski T, BreczewskiT,(1980), Dielectric and Pyroelectric properties of TGS crystal doped with nitroaniline molecules Ferrolelctric 25(1):547-550.

Ostrowski A, Bednarski W, Jesionowski T,(2012), Dielectric properties of fine grained Triglycine Sulphate(TGS), Journal of Non Crystalline Solid,35: 217- 219. 
Prasolov A.N., Palagin M.Y., and Gorbatenko V.V.(1998),Dielectric Hysteresis loop dispersion in TGS crystal Ferroelectrics, 214: 325-328.

Sun D,X,Yu,Q,Gu(1999) Growth Kinetics and Mechanics of TGS Crystal, Cryst. Re. Technol,34,1255.

Tello and Harnandez(1973), Phenomenological Parameters in TGS, Journal of Physical Society of Japan,35(5):1289-1291.

Yamaguchi T, Nakatani N, Toshio Kikuta, Kurihama T,(2006) Dilatometric study on monoclinic crystal of ferroelectric TGS down to cryogenic temperature region Ferrolectric 337(1): 59-69

Zubarev D.N.,(1960),Double time Green Function in Statistical physics Sov. Phys. Uspekhi, 3,320. 